\title{
8 Kostensensible Leitlinien als Rationierungs- instrumente in der GKV? Eine Beurteilung aus sozialrechtlicher Sicht
}

\author{
Ernst Hauck
}

\subsection{Das Problem}

Die Mittel im Gesundheitswesen sind knapp. Deshalb hat die Suche nach Einsparungen in medizinisch rationaler, ökonomisch sinnvoller und ethisch sowie rechtlich vertretbarer Weise Konjunktur. ${ }^{54}$ Eine Frucht dieser Mühen ist der Vorschlag des interdisziplinären, vom Bundesministerium für Bildung und Forschung finanzierten Forschungsverbundes „Allokation“, Leistungsbegrenzungen in der Gesetzlichen Krankenversicherung (GKV) durch Kostensensible Leitlinien einzuführen. Dieser Vorschlag soll im Folgenden kurz skizziert (s. Kap. 8.2) und einer methodenkritischen Bewertung (s. Kap. 8.3) unter Berücksichtigung von Regelungsalternativen (s. Kap. 8.4) unterzogen werden. Dies erlaubt mit der sich anschließenden rechtlichen Bewertung de lege lata (s. Kap. 8.5) und de lege ferenda (s. Kap. 8.6), Schlussfolgerungen zu ziehen (s. Kap. 8.7). Zur Vereinfachung wird für die folgende Diskussion die Prämisse als richtig unterstellt, weitere Leistungsbegrenzungen seien erforderlich, obwohl sich diese Annahme keinesfalls von selbst versteht. ${ }^{55}$

$54 \mathrm{Vgl}$. z.B. Stellungnahme der Zentralen Kommission zur Wahrung ethischer Grundsätze in der Medizin und ihren Grenzgebieten 2007; Wohlgemuth/Freitag (Hrsg.) 2009.

55 Zu dem unverändert nicht zu vernachlässigenden Ansatz, Effizienzreserven zu heben, vgl. z.B. Hauck 2008: $36 f$. 
8 Kostensensible Leitlinien als Rationierungsinstrumente in der GKV?

Eine Beurteilung aus sozialrechtlicher Sicht

\subsection{Kostensensible Leitlinien als Rationierungsinstrumente in der GKV}

Kostensensible Leitlinien zielen darauf ab, Leistungsbeschränkungen einzuführen. Patienten sollen auf solche Maßnahmen keinen Anspruch haben, die bei relativ hohen Kosten einen nur geringen Zusatznutzen bieten. Hierzu soll auf der Grundlage wissenschaftlicher Evidenz zur Effektivität und Kosteneffektivität insbesondere bei teuren Therapiemaßnahmen zunächst ermittelt werden, welche Gruppen und Untergruppen von Patienten, bei denen die Therapie indikationsgerecht einsetzbar ist, jeweils welchen Nutzen von der jeweiligen Therapiemaßnahme haben. In einem zweiten Schritt wird die Kosteneffektivität für die verschiedenen Patientengruppen bestimmt. Bei gleich bleibenden Behandlungskosten ist die Kosteneffektivität schlechter, je geringer der Nutzengewinn für die Patienten gegenüber der nächstgünstigeren Alternative ist. Die Kostensensible Leitlinie legt ausdrücklich den Grenzwert des Verhältnisses von Kosten zu Nutzen fest, bis zu dem die GKV die Therapiemaßnahme trägt. Übersteigt das Verhältnis von Kosten zum Nutzen den festgelegten Grenzwert, fällt die Therapiemaßnahme nicht mehr in den Leistungskatalog der GKV, sondern wird der Eigenverantwortung der Versicherten zugeordnet. Die Folge ist, dass versicherte Patienten mit einem großen Nutzengewinn die Therapiemaßnahme von der GKV erhalten, während versicherte Patienten, die von der Therapiemaßnahme nur wenig profitieren würden, auf eine kostengünstigere Behandlungsmöglichkeit ausweichen oder die Maßnahme privat finanzieren müssen.

Das Regelungsinstrument Kostensensibler Leitlinien leitet seine innere Rechtfertigung daraus ab, dass es sich um eine explizite Form der Leistungsbegrenzung „oberhalb“ der individuellen Arzt-Patienten-Beziehung nach ausdrücklich festgelegten, allgemein verbindlichen Kriterien handele. Dies sei gegenüber Formen impliziter Rationierung vorzugswürdig, die jeweils im Einzelfall durch Ärzte aufgrund begrenzter Budgets oder finanzieller Anreize wie der Fallpauschalenvergütung im Krankenhaus erfolge, wie eine Umfrage ${ }^{56}$ zeige. Zudem sollten Leistungsbegrenzungen in der GKV, wenn sie denn unausweichlich seien, so durchgeführt werden, dass Patienten ein möglichst geringer (Zusatz-)Nutzen vorenthalten werde.

\subsection{Methodenkritische Bewertung}

Kostensensible Leitlinien sollten als Regelungsinstrument expliziter Leistungsbegrenzung nur mit anderen expliziten Begrenzungsformen von GKV - Leistungen verglichen werden, nicht dagegen mit sog impliziten Formen der Leistungsbegrenzung. Die sog implizite Rationierung ${ }^{57}$ hat überhaupt nicht die Aufga-

$56 \mathrm{Vgl} \mathrm{dazu}$ Strech et al. 2009 m.w.N.

$57 \mathrm{Vgl}$. auch Huster et al. 2007 
be, Leistungsansprüche der Versicherten zu begrenzen, sondern eine andere, gerechtfertigte Funktion: Sie soll vorhandene, versteckte Effizienzreserven vor allem durch Vermeidung von Überflüssigem und Schaffung von Transparenz heben, ohne den Leistungsanspruch des Versicherten anzutasten. Rechtlich haben deshalb Regelungen wie die Budgetierung von Leistungen oder die Einführung von Fallpauschalen nichts am Leistungsanspruch des Versicherten geändert. Im Gegenteil zeigen die Vorkehrungen des Gesetzgebers wie etwa die Einführung von Stichprobenprüfungen, ${ }^{8}$ dass er Sicherungen vorgesehen hat, um einer rechtlich in keiner Weise gerechtfertigten Verkürzung der Ansprüche der Versicherten entgegen zu wirken. Die Prüfungen stellen sicher, dass etwa das ökonomische Eigeninteresse eines Krankenhausträgers, im Rahmen der Fallpauschalen die Verweildauer möglichst kurz zu halten, keine vorzeitigen („blutigen“) Entlassungen zeitigt. Ärzte, die sich ihren Rechtspflichten unter Hinweis auf sog implizite Leistungsbegrenzungen zu entziehen trachten, riskieren zu Recht straf-, haftungs-, berufs-, disziplinar- und zulassungsrechtliche Sanktionen.

Diese klare Rechtslage kann auch nicht durch Befragungen oder Statistiken über die Häufigkeit von Rechtsverstößen in Zweifel gezogen oder gar geändert werden: Ein solcher Versuch würde in unzulässiger Weise die Ebene des Sollens, des rechtlich Gebotenen, mit der Ebene des Seins vermischen, die die Beachtung der rechtlichen Gebote betrifft. Anders gewendet: Auch wer die Anreizwirkung der Gestaltung einer Auslage in einem Warenhaus anhand der daraufhin erfolgten Änderung der Zahl der Ladendiebstähle in diesem Kaufhaus misst, legitimiert damit keinen Diebstahl.

Aus diesen Überlegungen ergeben sich weitere Folgerungen für das methodische Vorgehen: Die eigentliche „Nagelprobe“ für die Qualität Kostensensibler Leitlinien als Regelungsinstrument zur Begrenzung von GKV-Leistungen ist der Vergleich mit anderen Regelungsinstrumenten ausdrücklicher Leistungsbegrenzung. Im Übrigen stellen sich bei der rechtlichen Bewertung vor allem zwei Fragen: Welche innere Struktur und welche Regelungsform von Leistungsbegrenzungen gebietet die Rechtsordnung oder lässt sie zu?

\subsection{Alternative Regelungsinstrumente zur Leistungsbegrenzung in der GKV}

Es würde den Rahmen dieser Untersuchung sprengen und hieße, die Kreativität anderer zu unterschätzen, wollte man auch nur den größten Teil denkbarer Alternativen zu Leistungsbegrenzungen durch Kostensensible Leitlinien darstellen. Es erschiene auch nicht als seriös, ganz überwiegend abgelehnte Al-

58 Vgl. § 17c Abs. 1 und 2 KHG, eingefügt durch Art. 2 Nr. 5 des Gesetzes vom 23.4.2002, BGBI I 1412, und hierzu BSG, Urteil vom 30.6.2009 - B 1 KR 24/08 R - Rn. 21ff., zur Veröffentlichung in BSGE und SozR vorgesehen. 
8 Kostensensible Leitlinien als Rationierungsinstrumente in der GKV?

Eine Beurteilung aus sozialrechtlicher Sicht

ternativen in den Blick zu nehmen wie etwa das Modell altersabhängiger Leistungsbudgetierung, ${ }^{59} \mathrm{um}$ die Idee einer Leistungsbegrenzung durch Kostensensible Leitlinien umso heller erstrahlen zu lassen. Aus praktischen Gründen genügt es, sich insoweit mit wesentlichen, bisher vom Gesetzgeber eingeschlagenen Wegen einer Leistungsbegrenzung auseinander zu setzen und diese mit Kostensensiblen Leitlinien zu vergleichen.

Der Gesetzgeber des SGB V ist bisher mehrspurig vorgegangen. Anders als beim Modell der Kostensensiblen Leitlinien, die ihrer Art nach ein Querschnittsinstrument für alle medizinischen Maßnahmen darstellen, hat er bisher einerseits Leistungsbeschränkungen bei Bagatellen und kernbereichsfremden Leistungen zu regeln gesucht. So hat er etwa für Maßnahmen der künstlichen Befruchtung statt voller nur eine hälftige Kostenübernahme eingeführt, ${ }^{60}$ grundsätzlich ${ }^{61}$ nicht verschreibungspflichtige Arzneimittel aus dem GKVLeistungskatalog ausgeschlossen, ${ }^{62}$ ebenso andere Arzneimittel, deren Kosten eher als dem Einzelnen tragbar erscheinen. ${ }^{63} \mathrm{Er}$ hat weiter das Sterbegeld zunächst beschränkt und dann ganz aus dem Leistungskatalog der GKV gestrichen ${ }^{64}$ und die Leistungen bei Zahnersatz eingeschränkt, ${ }^{65} \mathrm{um}$ nur einige Beispiele anzusprechen. Andrerseits hat er - eher mit Querschnittswirkung - im umfassenden Sinne Zuzahlungsregelungen zunächst eingeführt und später ausgeweitet, etwa die sog Praxisgebühr ${ }^{66}$ und Zuzahlungen für Arznei- und Verbandmittel, ${ }^{67}$ empfängnisverhütende Mittel, ${ }^{68}$ Medizinprodukte, ${ }^{69}$ Heilmittel, ${ }^{70} \mathrm{Hilfsmittel,},{ }^{71}$ häusliche Krankenpflege, ${ }^{72}$ Soziotherapie, ${ }^{73}$ Haushaltshilfe, ${ }^{74}$ stationären Maßnahmen ${ }^{75}$ sowie Fahrkosten. ${ }^{76}$

Die Rechtsprechung hat diese Konzeption der gesetzlichen Leistungsbeschränkungen bisher als verfassungskonform angesehen. Die gesetzlichen Krankenkassen sind nämlich weder nach dem SGB V noch von Verfassungs wegen ge-

59 Vgl. z.B. Breyer/Schultheiss 2003: 255ff.; Menzel 1996; Gutmann/Schmidt (Hrsg.) 2002; ablehnend z.B. Isensee 2005: 435; Kirchhof 2004: 3ff.; Neumann 2005: 622f.; Hauck 2007a: 208f. m.w.N.

60 Vgl. zur Verfassungsmäßigkeit BSG SozR 4-2500 \& 27a Nr. 5.

61 Zu Ausnahmen vgl. $\$ 34$ Abs. 1 Satz 2ff. SGB V.

62 Zur Verfassungsmäßigkeit und zur Vereinbarkeit mit europäischem Recht vgl. BSGE 102, $30=$ SozR 4-2500 § 34 Nr. 4.

63 Vgl. $\$ 34$ Abs. 1 S. 6 bis 9 SGB V.

64 Siehe näher BSG SozR 4-2500 \& 58 Nr. 1 m.w.N.

$65 \$ \$ 55 f f . ~ S G B V$ in den ab Inkrafttreten des GMG vom 14.11.2003, BGBI I 2190, geltenden Fassungen.

$66 § 28$ Abs. 4 SGB V.

$67 \oint 31$ Abs. 3 S. $1, \oint 23$ Abs. 3 SGB V.

$68 \int 24 a$ Abs. 2 Hs. 2 SGB V.

$69 \int 31$ Abs. 3 S. 1 i.V.m. Satz 3 SGB V.

$70 § 32$ Abs. 2 S. 1 SGB V.

$71 \oint 33$ Abs. 8S. 1 SGB V.

$72 \oint 37$ Abs. 5 SGB V.

$73 \bigcirc 37 a$ SGB V.

$74 \bigcirc 38$ Abs. 5 SGB V.

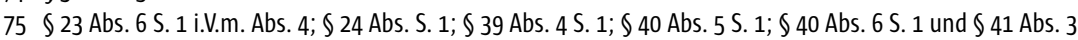
SGB V.

$76 § 60$ Abs. 1 S. 3 SGB V. 
halten, alles zu leisten, was an Mitteln zur Erhaltung oder Wiederherstellung der Gesundheit verfügbar ist. ${ }^{77}$ Der Leistungskatalog der CKV darf vielmehr auch von finanzwirtschaftlichen Erwägungen mitbestimmt sein. ${ }^{8}$ Cerade im Gesundheitswesen hat der Kostenaspekt für gesetzgeberische Entscheidungen erhebliches Gewicht. ${ }^{79}$ Dem Gesetzgeber ist es dabei im Rahmen seines Gestaltungsspielraums grundsätzlich auch erlaubt, den Versicherten über seinen Beitrag hinaus zur Entlastung der Krankenkassen und zur Stärkung des Kostenbewusstseins in der Form von Zuzahlungen zu bestimmten Leistungen zu beteiligen, jedenfalls, soweit dies dem Einzelnen finanziell zugemutet werden kann. ${ }^{80}$

Entsprechende Rechtsänderungen sind verfassungsrechtlich mit Wirkung für die Zukunft zulässig, sofern sie nicht verfassungsrechtlich geschütztes Vertrauen in die Aufrechterhaltung des zuvor geltenden Rechts verletzen, insbesondere nicht den Krankenversicherungsschutz insgesamt entwerten. ${ }^{81}$ Eine solche Entwertung kommt bei einer Änderung der Zuzahlungsregelungen nicht in Betracht, die den eigentlichen Kern der GKV-Leistungen nicht berührt. ${ }^{82}$

Die Regelungskonzeption der dargestellten, nach und nach in Kraft getretenen Leistungsbegrenzungen des Gesetzgebers zielt rechtspolitisch wesentlich darauf ab, der Gefahr einer Entsolidarisierung durch Zweiklassenmedizin entgegen zu treten, die in der Bundesrepublik Deutschland bisher erfolgreich vermieden worden ist. ${ }^{8}{ }_{3}$ Diese Gefahr birgt hingegen die Konzeption der Rationierung durch Kostensensible Leitlinien in sich. Denn sie schließt versicherte Patienten von notwendigen Therapiemaßnahmen unter Hinweis auf das bei ihnen ungünstige Kosten-Nutzen-Verhältnis aus, und dies gerade - so das Regelungsmodell - in relativ kostenteuren Segmenten, in denen Versicherte regelmäßig nicht in der Lage sind, sich die fehlenden Leistungen mithilfe eigener Mittel selbst zu verschaffen. Kostensensible Leitlinien erweitern die medizinische um eine ökonomische Indikation.

\subsection{Rechtliche Bewertung Kostensensibler Leitlinien de lege lata}

Auf der Crundlage des derzeit geltenden Gesetzesrechts können Kostensensible Leitlinien - ohne Gesetzesänderung - nicht wirksam in die deutsche

77 Vgl. z.B. BVerfGE 115, 25, 46 = SozR 4-2500 \$27 Nr. 5 Rn. 27; BVerfG, Beschluss der 2. Kammer des Ersten Senats, NJW 1997, 3085; BSGE 96, $153=$ SozR 4-2500 \& 27 Nr. 7, jeweils Rn. 28f. m.w.N.; BSGE 100, $103=$ SozR 4-2500 $\$ 31$ Nr. 9, Rn. 46 - Lorenzos Öl.

78 Vgl. BVerfGE 68, 193, $218=$ SozR 5495 Art. 5 Nr. 1; 70, 1, 26, $30=$ SozR 2200 \& 376d Nr. 1.

79 Vgl. BVerfGE 103, 172, $184=$ SozR 3-5520 \& 25 Nr. 4.

80 Vgl. BVerfGE 115, 25, 46 = SozR 4-2500 \& 27 Nr. 5 Rn. 27; BVerfG, Beschluss der 2. Kammer des Ersten Senats vom 7.3.1994 - 1 BvR 2158/93 -NJW 1994, 3007; BVerfGE 70, 1, 26, 30 = SozR $2200 \$ 376 d$ Nr. 1.

81 Vgl. z.B. BVerfGE 69, 272, 309f. = SozR $2200 \$ 165$ Nr. 81 S. 132; BSG SozR 4-2500 § 58 Nr. 1 Rn. 20f. m.w.N.

82 Vgl. BSGE 100, 221 = SozR 4-2500 \& 62 Nr. 6, jeweils Rn. 15 m.w.N.

83 Vgl. Hauck 2007c: 65 bei Fn. 19. 
8 Kostensensible Leitlinien als Rationierungsinstrumente in der GKV?

Eine Beurteilung aus sozialrechtlicher Sicht

Rechtsordnung eingeführt werden. Ihnen fehlte es schon an der erforderlichen formellen Legitimation des Normgebers.

Wer dazu berechtigt sein soll, Kostensensible Leitlinien zu erlassen, ist dem Modellvorhaben nicht klar zu entnehmen. Ohne Gesetzesänderung kommen mangels einer Ermächtigungsgrundlage für den Verordnungsgeber - denkmöglich - lediglich Zusammenschlüsse wie die wissenschaftlichen medizinischen Fachgesellschaften (s. Kap. 8.5.1) oder der Gemeinsame Bundesausschuss (G-BA) ${ }^{84}$ (s. Kap. 8.5.2) in Betracht. Die geltende Rechtsordnung beruft aber keines dieser Gremien dazu, Entscheidungen von der Tragweite Kostensensibler Leitlinien mit Wirkung für Versicherte, Beitragszahler, Krankenkassen und Leistungserbringer zu treffen.

\subsubsection{Wissenschaftliche medizinische Fachgesellschaften}

Nach der Rechtsprechung ist es für krankenversicherungsrechtliche Ansprüche der Versicherten ohne Belang, dass Leitlinien einer wissenschaftlichen Fachgesellschaft eine Behandlung als „Mittel der ersten Wahl“ ansehen. Grundsätzlich bestimmen nämlich nicht Leitlinien der medizinischen Fachgesellschaften den Umfang der Leistungsansprüche der Versicherten der CKV. Das Leistungsrecht ist vielmehr insbesondere von den Vorgaben der $\mathbb{2} 2 \mathrm{Abs} .1$ S. 1 und $3, \mathbb{S} 12$ SGB V geprägt, wonach Qualität und Wirksamkeit der Leistungen dem allgemein anerkannten Stand der medizinischen Erkenntnisse und dem Wirtschaftlichkeitsgebot entsprechen müssen. ${ }^{85}$ Den Fachgesellschaften kommt diesbezüglich keinerlei Rechtsetzungskompetenz zu. Allerdings ist es nicht ausgeschlossen, dass Leitlinien medizinischer Fachgesellschaften - je nach Design und Zielsetzung - zutreffend den allgemein anerkannten Stand der medizinischen Erkenntnisse widerspiegeln und deshalb zu dessen Ermittlung - wenn auch kritisch - heranzuziehen sind. ${ }^{86}$

Bei der Schaffung Kostensensibler Leitlinien geht es indes nicht allein um die Wiedergabe des allgemein anerkannten Standes der medizinischen Erkenntnisse. Dieser spielt vielmehr lediglich für Teilaspekte eine Rolle, nämlich für die Ermittlung, welche Gruppen und Untergruppen von Patienten, bei denen eine bestimmte Therapie indikationsgerecht einsetzbar ist, jeweils welchen Nutzen von der jeweiligen Therapiemaßnahme haben, die Gegenstand der Leitlinie werden soll. Im Übrigen geht es um die Kosteneffektivität der Therapiemaßnahme und vor allem um die normative Festlegung des maßgeblichen Grenzwertes, der den Anspruch der Versicherten begrenzen soll. Das Regelungssystem des SGB V sieht keine Ermächtigung der wissenschaftlichen

$84 \Im 91$ SGB V.

85 BSG, Urteil vom 30.6.2009, B 1 KR 5/09 R, SozR 4-2500 \& 31 Nr. 15, Rn. 47.

86 Vgl. für eine Auslandsbehandlung z.B. BSG SozR 4-2500 \& 18 Nr. 5 Rn. 33. 
Fachgesellschaften vor, solche Grenzwerte festzulegen, die Ansprüche der Versicherten begrenzen.

\subsubsection{Gemeinsamer Bundesausschuss}

Der G-BA beschließt nach der gesetzlichen Regelung des $₫ 92$ Abs. 1 Satz 1 SGB V die zur Sicherung der ärztlichen Versorgung erforderlichen Richtlinien über die Gewährung für eine ausreichende, zweckmäßige und wirtschaftliche Versorgung der Versicherten; dabei ist den besonderen Erfordernissen der Versorgung behinderter oder von Behinderung bedrohter Menschen und psychisch Kranker Rechnung zu tragen, vor allem bei den Leistungen zur Belastungserprobung und Arbeitstherapie; er kann dabei die Erbringung und Verordnung von Leistungen einschließlich Arzneimitteln oder Maßnahmen einschränken oder ausschließen, wenn nach allgemein anerkanntem Stand der medizinischen Erkenntnisse der diagnostische oder therapeutische Nutzen, die medizinische Notwendigkeit oder die Wirtschaftlichkeit nicht nachgewiesen sind sowie wenn insbesondere ein Arzneimittel unzweckmäßig oder eine andere, wirtschaftlichere Behandlungsmöglichkeit mit vergleichbarem diagnostischen oder therapeutischen Nutzen verfügbar ist. ${ }^{87}$

Auf dieser - allein in Betracht kommenden - Grundlage könnte der G-BA nur dann Kostensensible, Leistungsansprüche einschränkende Leitlinien im dargelegten Sinne mit Wirkung gegenüber Versicherten, Krankenkassen und Leistungserbringern ${ }^{88}$ erlassen, wenn die Überschreitung des in der Leitlinie gesetzten Grenzwertes damit gleichgesetzt werden könnte, dass der Nutzen, die medizinische Notwendigkeit oder die Wirtschaftlichkeit nicht nachgewiesen sind. Eine solche Auslegung des Gesetzes ist rechtlich ausgeschlossen.

Die Begriffe „Nutzen, medizinische Notwendigkeit und Wirtschaftlichkeit“ definiert das Gesetz nicht näher, auch wenn es sie vielfach sowohl im Rahmen der allgemeinen Vorschriften ${ }^{89}$ als auch im Dritten Kapitel „Leistungen der Krankenversicherung "90 sowie im Leistungserbringungsrecht ${ }^{91}$ und in Folgenormen ${ }^{92}$ verwendet. ${ }^{93}$ Soziologen könnten die quasi beschwörende ${ }^{94}$ Begriffshäufung als Indiz dafür ansehen, dass erhebliche Unwirtschaftlichkeit in

\footnotetext{
$87 \oint 92$ Abs. 1S. 1 SGB V.

88 Siehe $\$ 91$ Abs. 6 SGB V und hierzu BSG, Urteil vom 27.10.2009 - B 1 KR 4/09 R -, zur Veröffentlichung in BSGE und SozR vorgesehen.

89 SS 1 ff. SGB V.

90 \&S 11ff. SGB V.

91 Viertes Kapitel SGB V, $\$ \$ 69 f f$. SGB V.

92 SS $142 f f$. SGB V.

93 Außer in $\S 92$ Abs. 1 SGB V zB „Nutzen“ in $\$ \oint 34$ Abs. 3 S. 3 und Abs. 4; 35b Abs. 1; 116b; 135; 138; 139; 139a SGB V; „medizinische Notwendigkeit“ oder "notwendig“ etc. z.B. in $\$ \$ 2 ; 4 ; 11 ; 13 ; 23 ; 27 ; 31 ; 33 ; 35 ; 35 a ; 39 ; 40$; 43; 55; 56; 70; 71; 75a; 84; 106; 111; 112; 115a; 115c; 275 SGB V; „wirtschaftlich“ in 78 Normen, u.a. \$S 2, 4; 12; 31; 34; 35; 35a; 35b, 37; 40; 56; 63; 67; 68; 70-73a; 84; 87; 106; 106a; 109ff. SGB V.

$94 \mathrm{Vgl}$. Schewe 1989.
} 
8 Kostensensible Leitlinien als Rationierungsinstrumente in der GKV?

Eine Beurteilung aus sozialrechtlicher Sicht

einem weiten Sinne im GKV-System besteht. Der Zusammenhang mit dem umfassend geltenden, allgemeinen Wirtschaftlichkeitsgebot des $\mathbb{1} 12 \mathrm{Abs}$. 1 SGB V, das mit der Regelung in $\mathbb{7} 70$ Abs. 1 S. 2 SGB V95 und $₫ 72$ Abs. 2 SGB V korrespondiert, ${ }^{96}$ spricht für eine Synchronisierung der Begriffe. Das führt zugleich allerdings zu einem altbekannten Problem des allgemeinen Wirtschaftlichkeitsgebots: Schon die BSG-Rechtsprechung zur RVO verwies darauf, dass seine Einzelkomponenten in einem untrennbaren inneren Zusammenhang stehen. ${ }^{97}$ Das SGB V hat hieran nichts geändert. Die Folge ist, dass ein weites Begriffsverständnis bei einer Komponente zu einem engeren Verständnis bei den anderen führt, ohne dass das zugrunde liegende Normprogramm deshalb anders ausfallen müsste: Es erfolgt sozusagen nur unter einer anderen Überschrift.

\section{a) Der Begriff „Nutzen“}

Das allgemeine Wirtschaftlichkeitsgebot ${ }^{98}$ meint: Die Leistungen müssen ausreichend, zweckmäßig und wirtschaftlich sein; sie dürfen das Maß des Notwendigen nicht überschreiten. Leistungen, die nicht notwendig oder unwirtschaftlich sind, können Versicherte nicht beanspruchen, dürfen die Leistungserbringer nicht bewirken und die Krankenkassen nicht bewilligen. Diese Regelung erwähnt zwar die Notwendigkeit und Wirtschaftlichkeit, nicht aber ausdrücklich den „Nutzen“, sondern die Zweckmäßigkeit. Die Vermutung, dass der Nutzen einer Leistung ihre Zweckmäßigkeit belegt, bestätigt - im Rahmen der Festbetragsregelung $-\mathbb{} 35$ Abs. 1 b Satz 1 SGB V. ${ }^{99}$ Danach liegt eine therapeutische Verbesserung (nach $\mathbb{3} 35$ Abs. 1 S. 32 . Halbsatz und Abs. 1a S. 2 SGB V) vor, wenn das Arzneimittel einen therapierelevanten höheren Nutzen als andere Arzneimittel dieser Wirkstoffgruppe hat und deshalb als zweckmäßige Therapie regelmäßig oder auch für relevante Patientengruppen oder Indikationsbereiche den anderen Arzneimitteln dieser Gruppe vorzuziehen ist. Zwingend ist dieser Schluss wegen des Wortlauts von $\mathbb{s} 92 \mathrm{Abs}$. 1 S. 1 Halbsatz 3 SGB V indes nicht. Offen bleibt bei der Gesetzeslektüre auch, ob sich der Normgehalt der Schlüsselbegriffe „Nutzen“, „Notwendigkeit“ und „Wirtschaftlichkeit" wandelt, je nachdem, ob die generelle, G-BA-bezogene oder individuelle, auf den einzelnen Versicherten bezogene Ebene betroffen ist und wie sich die Begriffe zueinander verhalten.

Der hinter dem generellen Prüfverfahren durch den G-BA stehende Zweck, das Wirtschaftlichkeits- und Qualitätsgebot zu sichern, ${ }^{100}$ um die versicherten Patienten zu schützen und für einen effizienten Umgang mit den Mitteln der

95 IdF des Gesetzes vom 22.12.1999, BGBI I 2626.

96 Vgl. BSGE 81, 73, $77=$ SozR 3-2500 \& 92 Nr. 7 S. 52.

97 Vgl. BSGE 17, 79, 84; 19, 123, 128; 26, 16, 20.

$98 § 12$ Abs. 1 SGB V.

99 Eingefügt durch Gesetz vom 26.4.2006, BGBI I 984, in Kraft getreten ab 1.5.2006.

$100 \mathrm{Vgl}$ oben, bei Fn. 60. 
Beitragszahler zu sorgen, indiziert indes eine grundsätzliche Entsprechung des Bedeutungsgehalts der genannten Begriffstrias mit den aufgezeigten Komponenten des allgemeinen Wirtschaftlichkeitsgebots des $\mathbb{1} 12 \mathrm{Abs}$. 1 SGB V unter Berücksichtigung von dessen inhaltlicher Verschränkung mit dem Qualitätsgebot des $\mathbb{2}$ Abs. 1 S. 3 SGB V.

Entsprechend der „Zweckmäßigkeit“ meint „Nutzen“ einer Leistung danach ihre Eignung, die materiellen Leistungskriterien des SGB V zu erfüllen: So besteht ein „Nutzen“ einer konkreten Maßnahme zur Krankenbehandlung, wenn sie nach dem allgemein anerkannten Stand der medizinischen Erkenntnisse Wirkungen erwarten lässt, die - auch unter Abwägung der nach diesem Maßstab zu erwartenden Nebenwirkungen - zumutbar den gesetzlichen Behandlungszielen des $\$ 27$ Abs. 1 S. 1 SGB V in ihrer jeweiligen Wertigkeit ${ }^{101}$ dienen. Die Ausrichtung an den gesetzlichen Behandlungszielen beleuchtet beispielhaft $\mathbb{3}$ 35 Abs. 1 S. 4 SGB V ${ }^{102}$. Danach sollen beim Patienten-Nutzen insbesondere die Verbesserung des Gesundheitszustandes, eine Verkürzung der Krankheitsdauer, eine Verlängerung der Lebensdauer, eine Verringerung der Nebenwirkungen sowie eine Verbesserung der Lebensqualität berücksichtigt werden. Diese gesetzliche Erläuterung des Patienten-Nutzens ist zugleich inhaltlich auf die Behandlungsziele des $\$ 27$ Abs. 1 S. 1 SGB V beschränkt: So ist etwa mit „Verbesserung des Gesundheitszustandes und der Lebensqualität“ als Zielpunkt nicht „Wellness“ gemeint, sondern z.B. die Heilung der Krankheit, die Verhütung ihrer Verschlimmerung oder die Linderung der durch sie hervorgerufenen Beschwerden. Um „Nutzen“ zu ermitteln, sind mithin alle nach dem allgemein anerkannten Stand der medizinischen Erkenntnisse bei der Anwendung am Patienten zu erwartenden Wirkungen einer Maßnahme festzustellen und die - gesetzesbezogen - erwünschten gegenüber den unerwünschten Wirkungen hinsichtlich Erfolgs- und Schadenseintrittswahrscheinlichkeit sowie -umfang abzuwägen. Für die Zumutbarkeit unerwünschter Nebenwirkungen ist deren Risiko, also die Relation zwischen dem möglichen Schaden und seiner Eintrittswahrscheinlichkeit, ${ }^{103}$ in Abwägung mit der Gesamtsituation ${ }^{104}$ maßgeblich. Einzubeziehen ist, ob und ggf. welche Alternativen mit welchem Chancen- und Risikoprofil bestehen: Ist etwa bei einem anderen Behandlungsweg die Erfolgschance bei gleichem Risikoprofil und Indikationsbereich größer, fehlt es am gebotenen „Nutzen“. Die Behandlung ist (individuell und) generell nicht zweckmäßig. Der G-BA hat sie nicht zuzu-

101 Z.B.: Im Rahmen der „Heilung“ Vorrang einer kausalen vor einer symptomatischen Therapie; Vorrang der „Heilung“ vor "Diagnostik“ in dem Sinne, dass Diagnostik ohne möglich therapeutische Konsequenz nicht erfasst ist.

102 IdF durch Art. 1 Nr. 20 GKV-WSG vom 26.3.2007, BGBI I 378.

103 Vgl allgemein zB BVerwG DVBI 2006, 1524ff.; BSGE 93, 149ff.; BVerwGE 121, 257ff., Rn <juris> 21; speziell für die GKV: BSG, Urteil vom 7.11.2006 - B 1 KR 24/06 R - LITT, Rn. 25, zur Veröffentlichung vorgesehen; zum Ermittlungsproblem vgl zB IQWiG 2006: 11ff.

104 Vgl zB BSG SozR 4-2500 \31 Nr. 4 Rn. 40; BSG, Urteil vom 7.11.2006 - B 1 KR 24/06 R - LITT, Rn. 22, $25 f$. 
8 Kostensensible Leitlinien als Rationierungsinstrumente in der GKV?

Eine Beurteilung aus sozialrechtlicher Sicht

lassen oder auszuschließen. Von diesem Grundverständnis geht auch die Rechtsprechung des BSC aus. ${ }^{105}$

Danach kann der G-BA Kostensensible Leitlinien nicht unter Hinweis auf den fehlenden Nachweis des „Nutzens“ einer Therapiemaßnahme erlassen, weil es bei der Kostensensiblen Leitlinie neben der medizinischen zusätzlich um eine ökonomische Indikation geht. Entsprechendes gilt für den fehlenden Nachweis der medizinischen Notwendigkeit:

\section{b) Der Begriff „medizinische Notwendigkeit“}

Die Begrenzung auf das „medizinisch Notwendige“ beschränkt die Behandlung auf das Erforderliche, Ausreichende: Besteht etwa bei der Behandlung einer Krankheit ein nützlicher, insgesamt zweckmäßiger therapeutischer „Korridor“, der z.B. hinsichtlich Dosierung oder Therapiefrequenz Spielräume eröffnet, ist nur das untere, noch ausreichende, hinreichende Ausmaß der Behandlung notwendig. Ob „medizinische Notwendigkeit“ eine Versorgungslücke voraussetzt, hängt vom Verständnis dessen ab, was mit „Lücke“ gemeint ist. Geht es um die Einführung einer neuen, mit den bisher vorhandenen gleichwertigen, gleich geeigneten und gleich wirtschaftlichen Methode, begründet der Hinweis auf die bereits vorhandenen, zugelassenen Methoden unter Berücksichtigung der zu beachtenden Vielfalt der Leistungserbringer ${ }^{106}$ und des Individualisierungsgrundsatzes ${ }^{107}$ nicht automatisch stets das Fehlen der medizinischen Notwendigkeit der neuen Methode. Teilweise erfasst die Rechtsprechung unter dem Punkt „medizinische Notwendigkeit“ auch den Ausschluss reiner Befindlichkeitsstörungen und die Wahrscheinlichkeit eines Abklingens der Symptome ohne Intervention, ohne die Prüfung auf die Verhinderung eines Übermaßes unter Abwägung therapeutischer Alternativen zu reduzieren. ${ }^{108}$ Ein sachlich insgesamt abweichendes Prüfprogramm ist damit nicht intendiert, da diese Gesichtspunkte ansonsten beim „Nutzen“ erfasst werden.

Auch insoweit kann der G-BA Kostensensible Leitlinien nicht unter Hinweis auf den fehlenden Nachweis der medizinischen Notwendigkeit einer Therapiemaßnahme erlassen, weil es bei der Leitlinie - wie dargelegt - zusätzlich um eine ökonomische Indikation geht.

105 Vgl. z.B. 1. Senat, BSGE 97, $190=$ SozR 4-2500 § 27 Nr. 12, Urteil vom 7.11.2006 - B 1 KR 24/06 R - LITT, Rn. 31, auch unter Einbeziehung der Kriterien des $\$ 17$ Verf0 vom 20.9.2005, BAnz 2005 S 16998, geändert durch Beschluss vom 18.4.2006, BAnz 2006 S 4876; 3. Senat, BSGE 97, 133 = SozR 4-2500 \$139 Nr. 2 - Rn. 30ff.; 6. Senat, BSGE 96, 261 = SozR 4-2500\$92 Nr. 5 - Rn. 43.

106 Vgl. \$2 Abs. 3 S. 1 SGB V.

107 Vgl. $\$ 33$ SGBI.

108 Vgl. etwa das obiter dictum in BSGE 96, 261 = SozR 4-2500 \& 92 Nr. 5 Rn. 71 im Anschluss an Engelmann 2006 : 254. 


\section{c) Der Begriff „Wirtschaftlichkeit im engeren Sinne“}

Der Nachweis der Wirtschaftlichkeit bedingt jedenfalls den Beleg, dass bei Existenz verschiedener gleich zweckmäßiger und notwendiger Behandlungsmöglichkeiten die Kosten für den gleichen zu erwartenden Erfolg geringer oder zumindest nicht höher sind (Minimalprinzip). ${ }^{109}$ Speziell für die Bewertung des Kosten-Nutzen-Verhältnisses bei Arzneimitteln sieht $\$ 35 \mathrm{~b}$ Abs. 1 S. 4 SGB V ${ }^{110}$ vor, dass bei der wirtschaftlichen Bewertung auch die Angemessenheit und Zumutbarkeit einer Kostenübernahme durch die Versichertengemeinschaft angemessen berücksichtigt werden. Das wirft die Frage auf, ob bei der generellen Wirtschaftlichkeitsprüfung durch den G-BA jeder noch so geringe zu erwartende therapeutische oder z.B. diagnostische Vorteil beliebig hohe Mehrkosten rechtfertigt, solange es an einer kostengünstigeren Alternative fehlt. Fällt der Zusatznutzen einer neuen gegenüber einer kostengünstigeren, etablierten Methode nicht ins Gewicht, wird die etablierte Methode evtl. bereits ausreichend, die neue Methode mithin nicht erforderlich und unwirtschaftlich sein. Ist aber der Zusatznutzen mehr als nur geringfügig, stellt sich die Frage zugespitzt, ob eine Leistungspflicht der GKV vom G-BA wegen ganz erheblicher Mehrkosten abgelehnt werden kann. Ist er hierzu befugt, darf er auch Kostensensible Leitlinien erlassen.

Eine solche Befugnis ließe sich indes nur bejahen, wenn $\mathbb{3} 35 \mathrm{~b}$ Abs. 1 S. 4 SGB V in seiner speziellen Ausprägung Ausdruck eines allgemeinen Rechtsgedankens ist. Das erscheint im Hinblick auf seine Sonderstellung, ${ }^{111}$ die speziell vorgesehenen Sicherungen insbesondere bei Mitteln ohne Alternative ${ }^{112}$ und den auf Arzneimittel eingeschränkten Anwendungsbereich kaum vertretbar. Auch die Sonderregelung in $\mathbb{3} 35^{C} \mathrm{~S}$. 1 SGB V ${ }^{13}$ spricht dagegen. Allerdings hat die BSGRechtsprechung - etwa für Hilfsmittel - in Relation zu den Kosten zunächst einen von ihr jeweils definierten Mindestnutzen gefordert, ${ }^{114}$ um eine begründbare, angemessene Relation zwischen Kosten und Gebrauchsvorteil anzunehmen. Sie hat diese Prüfung später ${ }^{115}$ jedoch allenfalls erwogen, wenn der zusätzliche Gebrauchsvorteil des Hilfsmittels im Alltagsleben eher gering, die dafür anfallenden Kosten im Vergleich zu einem bisher als ausreichend angesehenen Versorgungsstandard dagegen als unverhältnismäßig hoch einzuschätzen sind. Sie ist demgegenüber für den Bereich ärztlicher Behandlung zunächst eher davon ausgegangen, dass bei alternativlosen Therapien die Kos-

109 Vgl. z.B. 1. Senat, BSGE 97, $190=$ SozR 4-2500 \& 27 Nr. 12, Rn. 26; 3. Senat, BSGE 97, $133=$ SozR 4-2500 § 139

Nr. 2 - Rn. 40; 6. Senat, BSGE 96, $261=$ SozR 4-2500 $\$ 92$ Nr. 5 - Rn. 70.

110 IdF durch das GKV-WSG vom 26.3.2007, BGBII 378.

$111 \oint 106$ Abs. 2a Nr. 4 SGB V betrifft bei genauerer Analyse ebenfalls lediglich das Minimalprinzip.

$112 \mathrm{Vgl} \S 31$ Abs. 2a S. 7 SGB V IdF durch Art. 1 Nr. 16a) GKV-WSG, aa0; entsprechend für die Festbetragsfestsetzung bei Hilfsmitteln BSG SozR 4-2500 \& 33 Nr. 1 Rn. 13 unter Hinweis auf BVerfG SozR 3-2500 \& 35 Nr. 2.

113 IdF durch Art. 1 Nr. 20a GKV-WSG, aaO.

114 Vgl. z.B. BSG SozR 3-2500 \& 33 Nrn. 4, 16, 34 (3.Senat); Nr. 28 (8. Senat).

115 Vgl. BSG SozR 3-2500 \& 33 Nr. 44, S. 250; daran anknüpfend BSG SozR $4-2500$ \& 33 Nr. 8 S. 55 , 
8 Kostensensible Leitlinien als Rationierungsinstrumente in der GKV?

Eine Beurteilung aus sozialrechtlicher Sicht

ten ohne Bedeutung sind, ${ }^{116}$ deutet nun aber im Bereich der Therapiehinweise für Arzneimittel eine Annäherung zur neueren Hilfsmittelrechtsprechung an, ${ }^{117}$ ohne von der bisherigen Linie - aus dem Bereich ärztlicher Behandlung bei Alternativlosigkeit abzurücken. ${ }^{118}$ Jenseits eines Bagatellbereichs kann aus der Rechtsprechung danach keine Interpretation abgeleitet werden, die die Befugnis des G-BA begründen könnte, gestützt allein auf das Wirtschaftlichkeitsgebot Kostensensible Leitlinien zu erlassen. Die Rechtsprechung ist in einer solchen Situation auch nicht zu einer abweichenden Auslegung berufen. ${ }^{119}$ Vielmehr handelte es sich um eine „wesentliche“ Entscheidung, die dem Gesetzgeber vorbehalten ist. ${ }^{120}$

Ohne besondere gesetzliche Ausgestaltung ${ }^{121}$ ist der G-BA mithin nicht befugt, im dargelegten Sinne medizinisch nützliche und notwendige Maßnahmen unter Berufung auf ihre fehlende „Wirtschaftlichkeit“ deshalb aus dem generellen GKV-Leistungskatalog auszuschließen, weil er die Relation zwischen dem Nutzen und den Kosten als ungünstig einschätzt. Ein so grundlegender „Paradigmenwechsel“" ${ }_{122}$ bedarf spezifischer gesetzlicher Normierung. ${ }^{123}$ Der Gesetzgeber des GKV-WSG geht dementsprechend in den Gesetzesmaterialien ${ }^{124}$ davon aus, dass bei fehlender therapeutischer Alternative der Anspruch des Versicherten auf die medizinisch notwendige und zweckmäßige Therapie zu gewährleisten ist. Der G-BA ist danach de lege lata nicht befugt, Kostensensible Leitlinien im dargelegten Sinne zu erlassen.

\subsection{Rechtliche Bewertung Kostensensibler Leitlinien de lege ferenda}

De lege ferenda stellen sich für den Gesetzgeber die Fragen nach den verfassungsrechtlichen Grenzen einer Sachregelung (s. Kap. 8.6.1) sowie für Zuständigkeiten zum Erlass Kostensensibler Leitlinien (s. Kap. 8.6.2).

\subsubsection{Verfassungsrechtlichen Grenzen einer Sachregelung}

Eine Begrenzung der Leistungsansprüche Versicherter darf - wie bereits dargelegt ${ }^{225}$ - von vornherein bei gleichbleibendem Beitragsniveau nicht den Krankenversicherungsschutz insgesamt so entwerten, dass geschütztes Vertrauen

\footnotetext{
116 Vgl. BSGE 78, 70, 89f. = SozR 3-2500 \& 92 Nr. 6 S. 48.

117 Vgl. BSGE 96, $261=$ SozR 4-2500 \$ 92 Nr. 5 - Rn. 75.

118 Vgl. BSGE 96, $261=$ SozR 4-2500 \$ 92 Nr. $5-$ Rn. 76.

119 Vgl. z.B. BVerfGE 106, $275=$ SozR 3-2500 $\$ 35$ Nr. 2, <juris> Rn. 140, zum Sachleistungsprinzip; Großer Senat BSGE 99, 111 = SozR 4-2500 \$39 Nr. 10 Rn. 26.

120 Vergleichbar den Ausführungen in BVerfGE 106, $275=$ SozR 3-2500 \& 35 Nr. 2, <juris> Rn. 140.

121 Zu den Grenzen siehe unter Kap. 8.6

122 Entsprechend Kuhn 1962.

123 Ebenso Engelmann 2007: 142 und 144.

124 Vgl. Bericht des Ausschusses für Gesundheit, BT-Drucks. 16/4247 S. 32 zu Nr. 16 ( $\$ 31$ Abs. 2a) Sätze 6 und 7.

$125 \mathrm{Vgl}$. oben bei Fn. 80 .
} 
enttäuscht wird. ${ }^{126}$ Spezielle Grenzen ergeben sich aus den Entscheidungen des Bundesverfassungsgerichts (BVerfG) vom 6.12.2005 $5^{127}$ und 17.12.2002 ${ }^{128}$ sowie aus dem Gleichheitssatz.

\section{a) Entscheidung des Bundesverfassungsgerichts vom 6.12.2005}

Der Beschluss des BVerfG vom 6.12.2005 beanstandet eine verfassungswidrige Auslegung im Grundsatz verfassungsgemäßer Vorschriften des SGB V durch das BSG. Er wendet sich deshalb unmittelbar nicht an den Gesetzgeber, sondern an die Normanwender. Trotzdem enthält er auch für den Gesetzgeber Hinweise, dass es verfassungsrechtlich zulässig ist, Regelungen zu treffen, wonach

- Leistungen in der GKV nur nach Maßgabe eines allgemeinen Leistungskatalogs und unter Beachtung des Wirtschaftlichkeitsgebots zu erbringen sind,

- die nähere Konkretisierung der gesetzlichen Leistungsverpflichtung vor allem den an der vertragsärztlichen Versorgung teilnehmenden Ärzten vorzubehalten ist,

- ein Verfahren vorgesehen ist, in dem neue Untersuchungs- und Behandlungsmethoden auf ihren diagnostischen und therapeutischen Nutzen sowie ihre medizinische Notwendigkeit und Wirtschaftlichkeit geprüft werden, um die „Anwendung dieser Methoden zu Lasten der GKV auf eine fachlich-medizinisch zuverlässige Grundlage zu stellen“ und wonach

- die GKV nicht verpflichtet ist, all das zu leisten, was überhaupt an Mitteln zur Erhaltung oder Wiederherstellung der Gesundheit verfügbar ist. ${ }^{129}$

Damit zeigt er zugleich einen Rahmen verfassungsrechtlich zulässiger Gestaltung auf, der auch für Gesetzesänderungen zur Einführung Kostensensibler Leitlinien nutzbar ist.

Soweit er eine verfassungskonforme Auslegung des bestehenden Gesetzesrechts fordert, sind dem BVerfG-Beschluss indes ebenfalls Fingerzeige für den Gesetzgeber zu entnehmen. Er stellt klar: Eine Leistungsverweigerung der Krankenkasse unter Berufung darauf, eine bestimmte neue ärztliche Behandlungsmethode im ambulanten Bereich sei im Rahmen der GKV ausgeschlossen, weil der zuständige G-BA diese noch nicht anerkannt oder sie sich zumindest in der Praxis und in der medizinischen Fachdiskussion noch nicht

126 Vgl. z.B. BVerfGE 69, 272, 309f. = SozR $2200 \$ 165$ Nr. 81 S. 132; BSG SozR 4-2500 § 58 Nr. 1 Rn. 20f. m.w.N.

127 BVerfGE $115,25=$ SozR $4-2500 \& 27$ Nr. 5 .

128 Vgl. BVerfGE 106, $275=$ SozR 3-2500 \$ 35 Nr. 2.

129 Vgl. näher Hauck 2007b: $1320 f f$. 
8 Kostensensible Leitlinien als Rationierungsinstrumente in der GKV?

Eine Beurteilung aus sozialrechtlicher Sicht

durchgesetzt hat, verstößt gegen das Grundgesetz, wenn folgende drei Voraussetzungen kumulativ erfüllt sind:

- Es liegt eine lebensbedrohliche oder regelmäßig tödlich verlaufende Erkrankung vor.

- Bezüglich dieser Krankheit steht eine allgemein anerkannte, medizinischem Standard entsprechende Behandlung nicht zur Verfügung.

- Bezüglich der beim Versicherten ärztlich angewandten (neuen, nicht allgemein anerkannten) Behandlungsmethode besteht eine „auf Indizien gestützte“, nicht ganz fernliegende Aussicht auf Heilung oder wenigstens auf eine spürbare positive Einwirkung auf den Krankheitsverlauf.

Diese Grundsätze, die das BSG auch auf zumindest wertungsmäßig damit vergleichbare Erkrankungen zu erstrecken erwägt, ${ }^{130}$ müssen auch de lege ferenda Beachtung finden, wenn es um die gesetzliche Einführung Kostensensibler Leitlinien geht. Wenn schon bei lebensbedrohlichen oder regelmäßig tödlich verlaufenden Erkrankungen, für die eine allgemein anerkannte, medizinischem Standard entsprechende Behandlung nicht zur Verfügung, die Anwendung von Behandlungsmethoden zu Lasten der GKV gefordert werden kann, für die eine „auf Indizien gestützte“, nicht ganz fernliegende Aussicht auf Heilung oder wenigstens auf eine spürbare positive Einwirkung auf den Krankheitsverlauf besteht, ist dies umso eher für medizinischem Standard entsprechende Behandlungsmethoden zu fordern, die nur über eine ökonomische Indikation ausgeschlossen werden sollen. Unter Berücksichtigung des Grundsatzes der Verhältnismäßigkeit spricht einiges dafür, diesen Crundgedanken im hier interessierenden Zusammenhang schon bei weniger gravierenden Erkrankungen eingreifen zu lassen.

\section{b) Entscheidungen des Bundesverfassungsgerichts vom 17.12.2002}

Die Festbetragsurteile des BVerfG vom 17.12.2002 akzentuieren die erforderliche Regelungsklarheit, die bei einer gesetzlichen Einführung Kostensensibler Leitlinien gefordert wäre. Weil de lege lata die Versicherten Anspruch auf eine in der Qualität gesicherte Vollversorgung durch Sachleistungen aus einer Pflichtversicherung haben, die durch Arbeitnehmer- und Arbeitgeberbeiträge solidarisch finanziert wird, bedarf es klarer gesetzliche Vorgaben, wenn dieses Gesamtsystem aufgegeben werden soll. Ohne solche klaren Regelungen müssen sich Versicherte - ebenso wie im Bereich der Festbeträge - nicht mit einer Teilkostenerstattung abspeisen lassen, wenn (zum Fest- oder Höchstbetrag) keine adäquate Versorgung zu erlangen ist. Der Gesetzgeber ist diesem Gedan-

130 Vgl. BSGE 96, 153 = SozR 4-2500 \& 27 Nr. 7, jeweils Rn. 31 - D-Ribose. Damit ist eine strengere Voraussetzung umschrieben, als sie etwa mit dem Erfordernis einer „schwerwiegenden“ Erkrankung für die Eröffnung des sog Off-Label-Use - dazu BSGE 89, $184=$ SozR 3-2500 \$ 31 Nr. 8 - Sandoglobulin - formuliert ist, vgl. BSG SozR 4-2500 \$ 31 Nr. 8 Rn. 17 - Mnesis; BSG SozR 4-2500 \$27 Nr. 10 Rn. 34 - neuropsychologische Therapie. 
ken bei Einführung der Höchstbetragsregelung für Arzneimittel gefolgt: Nach $\sqrt{ } 31$ Abs. 2a Satz 7 SGB V ${ }^{131}$ sind Arzneimittel, deren Kosteneffektivität erwiesen ist oder für die eine Kosten-Nutzen-Bewertung nur im Vergleich zur Nichtbehandlung erstellt werden kann, weil eine zweckmäßige Therapiealternative fehlt, von der Festsetzung eines Höchstbetrags auszunehmen. Denn bei fehlender therapeutischer Alternative ist der Anspruch des Versicherten auf die medizinisch notwendige und zweckmäßige Therapie zu gewährleisten. ${ }^{132}$

\section{c) Gleichheitssatz}

Zusätzliche Anforderungen erwachsen aus dem speziellen und allgemeinen Gleichheitssatz gemäß Art. 3 GG: Nach Abs. 3 S. 2 dieser Regelung darf niemand wegen seiner Behinderung benachteiligt werden. Gemäß Art. 3 Abs . 1 GG sind alle Menschen vor dem Gesetz gleich. Der Gesetzgeber muss deshalb in grundlegenden Fragen wie der Einführung einer ökonomischen Indikation im Zusammenhang mit Kostensensiblen Leitlinien nicht nur selbst eine so klare Regelung treffen, dass sie Verstöße gegen den Gleichheitssatz vermeidet, sondern auch so weit wie möglich vermeiden, durch Verwendung unbestimmter Rechtsbegriffe eine Benachteiligung behinderter Menschen zu ermöglichen.

Unter diesem Aspekt erschiene etwa die unkritische Übernahme des Begriffs „QALY“" ${ }_{133}$ für die Umschreibung des normativen Grenzwertes Kostensensibler Leitlinien bedenklich, obwohl dieser Begriff in der Gesundheitsökonomie für die Kosten-Nutzwert-Analyse scheinbar etabliert ist. ${ }^{134}$ Die Bedenken beruhen vor allem ${ }^{135}$ darauf, dass es schon keinen allgemein anerkannten Weg für die Bestimmung des im Begriff „QALY“ enthaltenen Qualitätsfaktors gibt ${ }^{136}$ und bereits die Methodenwahl zu unterschiedlichen Ergebnissen führt, die mit den verfassungsrechtlichen Anforderungen an die Bestimmtheit einer gesetzlichen Regelung über die Einführung Kostensensibler, leistungsbeschränkender Leitlinien unvereinbar sind. Auslegungsprobleme bei einer bloßen Bezugnahme auf den Begriff „QALY“ im Gesetz könnten folglich mit herkömmlichen

131 In der Fassung des GKV-WSG vom 26.3.2007, BGBI I 378.

132 Vgl. BT-Drucks. 16/4247 S: 31f. zu Nr. 16, zu den Sätzen 6 und 7.

133 Quality-Adjusted-Life-Year: Maß für die Messung der gesundheitsbezogenen Ergebnisse medizinischer Maßnahmen, das neben der Lebensverlängerung die Lebensqualität in dieser Zeitspanne erfassen will. Hierzu wird die Lebenserwartung mit einem Qualitätsindex (Faktor q) gewichtet mit den Grenzwerten 1 für völliges Wohlbefinden und o für Tod. Die gesundheitsbezogenen Effekte medizinischer Maßnahmen lassen sich danach durch Addition der qualitätsbewerteten Zeiten bestimmen. Das erlaubt den Vergleich ganz unterschiedlicher medizinischer Interventionen. Vgl. insgesamt hierzu z.B. Breyer/Zweifel/Kifmann 2003: 28ff.; Schlander 2006: 24f.; Schöffski/v.d. Schulenburg (Hrsg.) 2007; v.d. Schulenburg/Greiner 2007: $205 f f$.

134 Vgl. etwa Ausschuss für Gesundheitsökonomie im Verein für Socialpolitik und Deutsche Gesellschaft für Gesundheitsökonomie (2009)

135 Zum Problem der Verzerrungen der Bewertungen im Rahmen der "QALYs“ etwa im Hinblick auf behinderte Menschen oder Kinder vgl. z.B. Schlander 2006: 25; IQWiG 2006: 60ff.; Hauck 2007d.

136 Illustrativ etwa Drummond et al. 2009. 
8 Kostensensible Leitlinien als Rationierungsinstrumente in der GKV?

Eine Beurteilung aus sozialrechtlicher Sicht

juristischen Methoden nicht bewältigt werden. ${ }^{137}$ Dem lässt sich nicht überzeugend entgegenhalten, der Gesetzgeber habe bereits verfassungskonform über die Rezeption von „QALYs“ in $\mathbb{3} 35$ b Abs. 1 S. 5 SGB V entschieden.

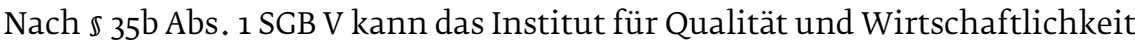
im Gesundheitswesen (IQWiC) ${ }^{138}$ beauftragt werden, den Nutzen oder das Kosten-Nutzen-Verhältnis von Arzneimitteln zu bewerten. Bewertungen nach Satz 1 können für jedes erstmals verordnungsfähige Arzneimittel mit patentgeschützten Wirkstoffen sowie für andere Arzneimittel, die von Bedeutung sind, erstellt werden. Die Bewertung erfolgt durch Vergleich mit anderen Arzneimitteln und Behandlungsformen unter Berücksichtigung des therapeutischen Zusatznutzens für die Patienten im Verhältnis zu den Kosten. Beim Patienten-Nutzen sollen insbesondere die Verbesserung des Gesundheitszustandes, eine Verkürzung der Krankheitsdauer, eine Verlängerung der Lebensdauer, eine Verringerung der Nebenwirkungen sowie eine Verbesserung der Lebensqualität, bei der wirtschaftlichen Bewertung auch die Angemessenheit und Zumutbarkeit einer Kostenübernahme durch die Versichertengemeinschaft, angemessen berücksichtigt werden. Das Institut bestimmt auftragsbezogen über die Methoden und Kriterien für die Erarbeitung von Bewertungen nach Satz 1 auf der Grundlage der in den jeweiligen Fachkreisen anerkannten internationalen Standards der evidenzbasierten Medizin und der Gesundheitsökonomie.

Bei der Anwendung des $\mathbb{3} 35 \mathrm{~b}$ Abs. 1 SGB V geht es schon nicht um einen Leistungsausschluss - wie es bei Kostensensiblen Leitlinien der Fall wäre -, sondern um die Vorbereitung einer Entscheidung über die Höchstbetragsfestsetzung für die betroffenen Arzneimittel, ${ }^{339}$ die allenfalls den Umfang der Kostentragung durch die Krankenkassen begrenzt. Hinzu kommt, dass das Gesetz selber Vorgaben für beim Patienten-Nutzen und bei der wirtschaftlichen Bewertung zu berücksichtigende Parameter macht, die nicht etwa durch vorgebliche ökonomische Standards überspielt werden dürfen. Vielmehr hat das IQWiG insoweit zunächst die gesetzlichen Wertungen zugrunde zu legen und sodann jeweils auftragsbezogen über die Methoden und Kriterien zu bestimmen für die Erarbeitung der relevanten Bewertungen auf der Crundlage der in den jeweiligen Fachkreisen anerkannten internationalen Standards u.a. auch der Gesundheitsökonomie. Damit lässt es das Gesetz nicht zu, seine eigenen Festlegungen durch Standards der Gesundheitsökonomie zu ersetzen, sondern eröffnet lediglich Spielräume, um im Rahmen der gesetzlichen Vorgaben Methoden und Kriterien für die Erarbeitung von Bewertungen - soweit dies danach überhaupt möglich und sachgerecht ist - auf der Crundlage anerkannter internationaler Standards zu bestimmen: Kollidiert ein anerkann-

137 Vgl. zur Maßgeblichkeit dieses Maßstabs z.B. BVerfGE 82, 209, 224ff.; 110, 370, 396f. m.w.N.

138 Nach $\oint 139 b$ Abs. 1 und 2 SGB V.

139 Vgl. $\$ 31$ Abs. 2a SGB V. 
ter internationaler Standard mit den Vorgaben des SGB V, ist er insoweit für die Aufgabe des IQWiG nach $\mathbb{3} 35$ b Abs. 1 S. 5 SGB V unbrauchbar.

\subsubsection{Verfassungsrechtliche Vorgaben für die Zuständigkeit zum Erlass Kostensensibler Leitlinien}

Aus dem oben ${ }^{140}$ Dargelegten folgt zunächst, dass der Gesetzgeber die wesentlichen Entscheidungen bei Einführung Kostensensibler Leitlinien selbst treffen müsste. Er wäre dadurch zwar nicht gehindert, die konkrete ökonomische Grenzwertbestimmung auf den Verordnungsgeber zu übertragen, ${ }^{141}$ wohl aber bestünden Bedenken gegen eine Übertragung dieser Aufgabe auf den G-BA.

Zwar sehen alle hierfür zuständigen Senate des BSG die - inzwischen auch gesetzlich normierte - Drittwirkung von Richtlinien des G-BA als verfassungsgemäß an. ${ }^{142}$ Nach der Rechtsprechung des BVerfG ${ }^{143}$ darf der parlamentarische Gesetzgeber nämlich Organe von Trägern der funktionalen Selbstverwaltung in begrenztem Umfang auch zu einem Handeln gegenüber Nichtmitgliedern ermächtigen. Die Voraussetzungen, dass Aufgaben und Handlungsbefugnisse der Organe in einem von der Volksvertretung beschlossenen Gesetz ausreichend vorher bestimmt sind und ihre Wahrnehmung der Aufsicht personell demokratisch legitimierter Amtswalter unterliegt, sind erfüllt durch die Regelungen im SGB V über den Erlass von Richtlinien durch den G-BA und seine Kontrolle. Es kommt hinzu, dass nach der gesetzlichen Konzeption - im wesentlichen - alle betroffenen Gruppen im Rahmen „gemeinsamer Selbstverwaltung“ einbezogen sind, den relevanten Regelungen im SGB V ein Art. 80 Abs. 1 Satz 2 GG genügendes Normprogramm zugrunde liegt ${ }^{144}$ und sich die spezifische Form der Regelung u.a. aus der Notwendigkeit rechtfertigt, den medizinischen Fortschritt zeitgerecht in die betroffenen Regelungen einzubeziehen. ${ }^{145}$

Diese Grundposition lässt sich aber nicht einfach auf die Eingrenzung der medizinischen durch die ökonomische Indikation durch Kostensensible Leitlinien übertragen. Während sich in den Leitlinien etwa bei Feststellung des jeweils relevanten Patientennutzens der „galoppierende“ medizinische Fortschritt auswirken kann, entspricht dem weder bei der Bestimmung der Kosteneffektivität noch bei der Festlegung des Grenzwertes des Verhältnisses von Kosten

140 Unter Kap. 8.5.2

141 Unter Beachtung der Vorgaben des Art. 80 GG.

142 Vgl. z.B. 1. Senat, Urteil vom 27.10.2009 - B 1 KR 4/09 R -, zur Veröffentlichung in BSGE und SozR vorgesehen; implizit 3. Senat in BSG, Urteil vom 12.8.2009 - B 3 KR 10/07 R -, zur Veröffentlichung in BSGE und SozR vorgesehen; 6. Senat, BSGE 96, $261=$ SozR 4-2500 \$92 Nr. 5, jeweils Rn. 57ff.; zusammenfassend Engelmann 2006: 248; kritisch Z.B. Kingreen 2006: 879, alle m.w.N.

143 Vgl. BVerfGE 107, 59, 94.

144 Vgl. hierzu auch BVerfGE 37, 1, 24ff. m.w.N.

145 Vgl. z.B. BSGE 78, $70=$ SozR 3-2500 \& 92 Nr. 6 <juris> Rn. 37. 
8 Kostensensible Leitlinien als Rationierungsinstrumente in der GKV?

Eine Beurteilung aus sozialrechtlicher Sicht

zu Nutzen ein ähnlich dynamischer Fortschritt der Ökonomie. Insoweit rechtfertigt sich nur für einen Teilaspekt der Regelung die Überantwortung an einen Normgeber, der im Vergleich zum Gesetz- oder Verordnungsgeber zu schnellerer Anpassung einer Leitlinie in der Lage ist. Nur insoweit würde auch an die herkömmliche Struktur der Richtlinien des G-BA angeknüpft, gesetzliche Crundentscheidungen zum (für Kostensensible Leitlinien gesetzlich zu modifizierenden) Wirtschaftlichkeitsgebot dem sich laufend änderndem Stand der medizinischen Erkenntnisse anzupassen.

\subsection{Schlussfolgerungen}

Das Regelungsmodell der Kostensensiblen Leitlinien bereichert das Diskussionsspektrum über explizite Begrenzungen der GKV-Leistungen. Das Regelungsmodell Kostensensibler Leitlinien wirkt auf den zweiten Blick allerdings weder ethisch noch rechtlich uneingeschränkt überzeugend. Insbesondere erscheint es als den bisherigen Ansätzen des Gesetzgebers unterlegen, Beschränkungen der GKV-Leistungen außerhalb von Kernleistungen in „Bagatellbereichen "vorzunehmen, die eher der Eigenvorsorge der Versicherten überantwortet werden können. Denkbar ist danach der Einsatz Kostensensibler Leitlinien in Bagatell-Grenzbereichen außerhalb von Kernleistungen der GKV im Interesse verhältnismäßiger Lösungen unter der Voraussetzung, dass hierfür die unabdingbar erforderliche solide gesetzliche Grundlage geschaffen wird. In diesem Sinne können Kostensensible Leitlinien das vorhandene Instrumentarium expliziter Leistungsbegrenzung nicht etwa ersetzen, sondern allenfalls ergänzen. Vorzugswürdig bleiben Ansätze, die eine Priorisierung von GKV-Leistungen vermeiden, etwa indem sie Effizienzreserven heben.

Dieser Beitrag ist zuerst im Jahr 2010 in Die Sozialgerichtsbarkeit 57, 193-200 erschienen. Mit freundlicher Genehmigung der Erich Schmidt Verlag GmbH \& Co. KG.

\section{Literatur}

Ausschuss für Gesundheitsökonomie im Verein für Socialpolitik und Deutsche Gesellschaft für Gesundheitsökonomie (2009) Stellungnahme zum „Entwurf einer Methodik für die Bewertung von Verhältnissen zwischen Nutzen und Kosten im System der deutschen gesetzlichen Krankenversicherung Version 2.0". URL: https://www.iqwig.de/download/Dokumentation_der_Stellungnahmen_KNB-Methodenentwurf_2.o.pdf (Zugriff am 21.04.2015)

Breyer F, Schultheiss C (2003) „Primary“ Rationing of Health Services in Ageing Societies - A Normative Analysis. Internat. Journ. of Health Care, Finance and Economics 2, 247-264

Breyer F, Zweifel P, Kifmann M (2003) Gesundheitsökonomie. 4. Aufl. Springer-Verlag: Berlin Heidelberg Drummond M, Brixner D, Gold M et al. (2009) Toward a Consensus on the QALY. Value Health 12, 31-35 Engelmann K (2007) Der Anspruch auf Krankenbehandlung im Hinblick auf das Wirtschaftlichkeitsgebot. In: Seewald 0 et al. (Hrsg.) Grenzen der Leistungspflicht für Krankenbehandlung 109-146. Manz: Wien 
Engelmann K (2006) Die Kontrolle medizinischer Standards durch die Sozialgerichtsbarkeit. Medizinrecht, 245-259

Gutmann T, Schmidt VH (Hrsg.) (2002) Rationierung und Allokation im Gesundheitswesen. Velbrück Wissenschaft: Weilerswist

Hauck E (2008) GKV - quo vadis? In: 1. Deutscher Sozialgerichtstag. Plan B - Solidarität neu denken. 33-49. Nomos: Baden-Baden

Hauck E (2007a) Entwicklungsperspektiven der gesetzlichen Krankenversicherung (GKV). Die Sozialgerichtsbarkeit, 203-209

Hauck E (2007b) Gestaltung des Leistungsrechts der gesetzlichen Krankenversicherung durch das Grundgesetz? Neue Juristische Wochenschrift: 1320-1325

Hauck E (2007c) Sozialrecht als Ort für einen geregelten Kassenwettbewerb? Recht und Politik im Gesundheitswesen $13,64-70$

Hauck E (2007d) Die Rechtsverfassung der Bewertung von Leistungen durch den Gemeinsamen Bundesausschuss (G-BA) und das Institut für Qualität und Wirtschaftlichkeit im Gesundheitswesen (IQWiG). Bewertungskriterien und -methoden nach dem SGB V. Vortrag vom 26.4.2007

Huster S, Strech D, Marckmann G, Freyer D, Börchers K, Neumann A, Wasem J, Held C (2007) Implizite Rationierung als Rechtsproblem. Ergebnisse einer qualitativen Interviewstudie zur Situation in deutschen Krankenhäusern. Medizinrecht, 703-706

IQWiG (2006) Methoden. Version 2.0 vom 19.12.2006

Isensee I (2005) Verwaltung des Mangels im Gesundheitswesen. In: Söllner A, Gitter W, Waltermann R, Giesen R, Ricken 0 (Hrsg.) Gedächtnisschrift für Meinhard Heinze. 417-435. Beck: München

Kingreen T (2006) Verfassungsrechtliche Grenzen der Rechtsetzungsbefugnis des Gemeinsamen Bundesausschusses im Gesundheitsrecht. Neue Juristische Wochenschrift, 877-880

Kirchhof P (2004) Das Recht auf Gesundheit. Stimmen der Zeit 1, 3-14

Kuhn TS (1962) The Structure of Scientific Revolutions. University of Chicago Press: Chicago

Menzel PT (1996) The Justification and Implications of Age-Influenced Rationing. In: Walters I (Hrsg.) Choosing Who's to Live: Ethics and Aging. 3-23. University of Illinois Press: Urbana

Neumann V (2005) Prioritätensetzung und Rationierung in der gesetzlichen Krankenversicherung. Neue Zeitschrift für Sozialrecht, 617-623

Schewe D (1989) Die Beschwörung des Wirtschaftlichen im neuen SGB V. Die Sozialgerichtsbarkeit, 410-411

Schlander M (2006) Was genau ist eigentlich ein QALY? Der Kassenarzt 7, 24-25

Schöffski 0, v.d. Schulenburg I-M (Hrsg.) (2007) Gesundheitsökonomische Evaluationen. 3. Aufl. Springer-Verlag: Berlin Heidelberg

v.d. Schulenburg I-M, Greiner W (2007) Gesundheitsökonomik. 2. Aufl. Mohr Siebeck: Tübingen

Strech D, Danis M, Löb M, Marckmann G (2009) Ausmaß und Auswirkungen von Rationierung in deutschen Krankenhäusern. Deutsche Medizinische Wochenschrift 134, 1261-1266

Wohlgemuth WA, Freitag MH (Hrsg.) (2009) Priorisierung in der Medizin - Interdisziplinäre Forschungsansätze. Medizinisch Wissenschaftliche Verlagsgesellschaft: Berlin

Zentrale Kommission zur Wahrung ethischer Grundsätze in der Medizin und ihren Grenzgebieten (Zentrale Ethikkommission) bei der Bundesärztekammer (2007) Stellungnahme zur Priorisierung medizinischer Leistungen im System der Gesetzlichen Krankenversicherung. URL: http://www.zentrale-ethikkommission.de/ downloads/LangfassungPriorisierung.pdf (Zugriff am 21.04.2015) 